La Revista Panamericana de Salud Pública/Pan American Journal of Public Health se complace en publicar cartas de los lectores dirigidas a estimular el diálogo sobre los diversos aspectos de la salud pública en las Américas, así como a esclarecer, discutir o comentar de manera constructiva las ideas expuestas en la revista. Las cartas deben estar firmadas por el autor y especificar su afiliación profesional y dirección postal o electrónica. Cuando se trate de comentarios sobre un artículo que requieren contestación del autor, se procurará conseguir esa respuesta con el fin de publicar ambas cartas. La Redacción se reserva el derecho de editar las cartas recibidas y resumirlas para mejorar su claridad.

The Revista Panamericana de Salud Pública/Pan American Journal of Public Health publishes letters from readers for the purpose of stimulating dialogue on various aspects of public health in the Americas and of constructively clarifying, discussing, and critiquing the ideas expressed throughout its pages. Letters should be signed by the author and include his or her professional affiliation and mailing or E-mail address. If a commentary on a given article requires a reply from the author, an effort will be made to obtain the reply and to publish both letters. The editorial team reserves the right to edit all letters received and to condense them so as to improve their clarity.

\section{REFORMAS DE SALUD NEOLIBERALES EN MÉXICO}

Editor: En el número 3, vol. 17 de la Revista Panamericana de Salud Pública publicado en marzo del presente año se presentó el artículo "Las reformas de salud neoliberales en América Latina: una visión crítica a través de dos estudios de caso", escrito por Nuria Homedes y Antonio Ugalde. En este artículo se describen elementos de la reciente reforma del sector de la salud en México como parte de un análisis sobre "las experiencias de América Latina relacionadas con la descentralización y la privatización de los servicios de salud". Al respecto, se han identificado imprecisiones que lejos de informar al lector, pueden generar una interpretación incompleta o incorrecta sobre los fundamentos de la reciente reforma en México.

A continuación se mencionan de manera breve las características del Seguro Popular en México y se abordan las imprecisiones mencionadas.

En 2003 el Congreso de la Unión aprobó un conjunto de reformas a la Ley General de Salud (LGS), mediante las cuales se introdujo un nuevo esquema de aseguramiento público de salud, de carácter voluntario y dirigido a la población sin acceso a la seguridad social, que plantea la definición explícita de los beneficios e intervenciones cubiertas. Esta reforma busca aumentar la inversión en la salud y ofrecer igualdad de oportunidades para todos los mexicanos en materia de protección financiera para la salud, independientemente de su ingreso, lugar de residencia, origen étnico o situación laboral, así como sentar las bases para eliminar la segmentación por grupos poblacionales que caracteriza hoy en día a nuestro sistema de salud.

En primer término, el artículo menciona que “... el Seguro Popular surgió en 2004 como un programa vertical..." Cabe aclarar que el Seguro Popular existe desde 2001. En ese año comenzó como programa piloto, contando con recursos asignados por el Congreso por un monto de 25 millones de dólares estadounidenses (US\$) en cinco entidades federativas - Aguascalientes, Campeche, Colima, Jalisco y Tabasco-, a las que se sumaron rápidamente más entidades interesadas en el programa. En 2003, 24 entidades se habían incorporado al Seguro Popular, alcanzándose una cobertura de 614000 familias afiliadas y contando con una asignación presupuestaria de US\$29,3 millones. Con la entrada en vigor de la reforma a la LGS el $1^{\circ}$ de enero de 2004, el Seguro Popular dejó de ser un programa piloto y se convirtió en el vehículo operativo del Sistema de Protección Social en Salud (SPSS).

Una segunda imprecisión se refiere a que “. . al dejar de percibir las cuotas, las secretarías estatales pierden la fuente más importante de ingre- 
sos. .." Si bien las cuotas de recuperación son una fuente de recursos en el ámbito estatal, en 2003 estas apenas representaron 5,3\% del total de los recursos con que cuentan las entidades federativas para financiar los servicios de salud dirigidos a la población sin seguridad social. Asimismo, lejos de mermar las finanzas de los servicios estatales de salud, estos se benefician con la introducción de un esquema de financiamiento tripartito conformado por recursos del gobierno federal (mediante una cuota social y una aportación solidaria federal), del gobierno estatal (representado por una aportación solidaria estatal) y de las familias beneficiarias (que se traduce en una cuota familiar). De hecho, se estima que una vez cubierta toda la población que no cuenta con seguridad social, los recursos públicos adicionales para el sistema de salud representarán alrededor de $1 \%$ del producto interno bruto. Cabe mencionar que bajo este esquema las aportaciones federales y estatales constituyen, por mucho, la principal fuente del financiamiento del sistema. De hecho, el presupuesto asignado al Seguro Popular se ha incrementado de manera muy notable tan solo en 2004 y 2005, registrando alrededor de US\$ 380 millones y US\$ 860 millones, respectivamente.

Además, es importante señalar que las cuotas de recuperación son también un gasto de bolsillo, y como tal, representan una forma poco equitativa e ineficiente de financiar la atención médica, con la única diferencia de que estas cuotas se pagan a las instituciones públicas que prestan servicios de salud. Esta vía de financiamiento es incompatible con la solidaridad financiera que permite que las personas enfermas y de escasos ingresos se beneficien de las contribuciones de las personas sanas y de mayores recursos, así como con el principio básico de justicia financiera en materia de salud, que establece que los individuos deben aportar de acuerdo con su capacidad de pago y recibir atención médica en función de sus necesidades de salud. El SPSS promueve la protección financiera para la salud al sustituir el gasto de bolsillo (donde se incluyen las cuotas de recuperación) por un seguro público (sustentado en los impuestos generales, que conforman la principal fuente de financiamiento). Con esto se busca crear una cultura de prevención mediante el aseguramiento en donde se perciba la conveniencia de asegurarse en vez de realizar pagos directos de bolsillo en el momento de requerir los servicios y, con ello, eliminar el riesgo de incurrir en gastos que empobrezcan a las familias mexicanas.

El tercer argumento se refiere a que ". . . la aplicación del seguro popular lleve, de hecho, a una centralización del sector de la salud. .." Al respecto, la reforma no implica una centralización del sector de la salud, como aseguran los autores. Por el contrario, la creación del SPSS da continuidad al proceso de descentralización que la Secretaría de Salud ha llevado a cabo desde la década de los ochenta. De hecho, la reforma a la LGS define con mayor claridad las atribuciones entre órdenes de gobierno y refuerza la prestación de servicios en el nivel estatal al incorporar de manera explícita la figura de los Regímenes Estatales de Protección Social en Salud, que son responsables de realizar las acciones de protección social en los estados. Adicionalmente, la reforma fortalece el proceso de descentralización al incorporar reglas que definen explícitamente los derechos y obligaciones para la aportación y concurrencia de recursos públicos para la salud. Igualmente, se refuerza este proceso con la rendición de cuentas para garantizar una mayor equidad en la prestación de los servicios y que el uso de los recursos corresponda a las necesidades de salud de la ciudadanía.

Como último punto, los autores mencionan que "... se promueve que los estados contraten en el sector privado servicios médicos cubiertos por el seguro popular. .." Al contrario, la reforma busca fortalecer las redes públicas de prestadores de servicios de salud. De hecho, el SPSS contempla un Plan Maestro de Infraestructura (PMI) (artículo 77 Bis 10 de la LGS), cuyo objetivo es incrementar la inversión financiera en infraestructura y equipamiento, sobre la base de un proceso de priorización de obras, para cubrir las necesidades de salud insatisfechas.

Por otra parte, como complemento al esfuerzo realizado por los autores para analizar la experiencia de la reforma en el caso de México, se sugiere consultar el documento Estudios de la OCDE sobre los sistemas de salud: México", publicado por la Organización para la Cooperación y el Desarrollo Económicos (OCDE), con sede en París, Francia, y presentado el pasado 6 de abril. Para obtener mayor información sobre esta publicación, se sugiere consultar la página de Internet del Centro de la OCDE en México para América Latina (www.ocdemexico.org.mx).

Finalmente, es importante resaltar que la precisión y objetividad de un análisis sobre el Sistema de Salud en México, señalando sus debilidades y reconociendo sus fortalezas, fomenta el debate imparcial y promueve una crítica constructiva que suma elementos para promover un mejor desempeño del sistema de salud. Esperamos que estos comentarios contribuyan a informar mejor el debate de las ideas sobre las políticas de salud de América Latina.

Dr. Eduardo González Pier

Titular de la Unidad de Análisis Económico Secretaría de Salud de México epier@salud.gob.mx 